\title{
Automated detection of Pi 2 pulsations using wavelet analysis: 1. Method and an application for substorm monitoring
}

\author{
M. Nosé ${ }^{1}$, T. Iyemori ${ }^{1}$, M. Takeda ${ }^{1}$, T. Kamei ${ }^{1}$, D. K. Milling ${ }^{2}$, D. Orr ${ }^{2}$, H. J. Singer ${ }^{3}$, E. W. Worthington ${ }^{4}$, and N. Sumitomo ${ }^{5}$ \\ ${ }^{1}$ Faculty of Science, Kyoto University, Kyoto 606-8502, Japan \\ ${ }^{2}$ Department of Physics, University of York, York Y010 5DD, U.K. \\ ${ }^{3}$ NOAA, Space Environment Center, Boulder, CO 80303, U.S.A. \\ ${ }^{4}$ College Observatory, U.S. Geological Survey, Fairbanks, AK 99775, U.S.A. \\ ${ }^{5}$ Disaster Prevention Research Institute, Kyoto University, Uji, Kyoto 611-0011, Japan
}

(Received December 9, 1997; Revised April 20, 1998; Accepted May 18, 1998)

\begin{abstract}
Wavelet analysis is suitable for investigating waves, such as Pi 2 pulsations, which are limited in both time and frequency. We have developed an algorithm to detect Pi 2 pulsations by wavelet analysis. We tested the algorithm and found that the results of Pi 2 detection are consistent with those obtained by visual inspection. The algorithm is applied in a project which aims at the nowcasting of substorm onsets. In this project we use real-time geomagnetic field data, with a sampling rate of 1 second, obtained at mid- and low-latitude stations (Mineyama in Japan, the York SAMNET station in the U.K., and Boulder in the U.S.). These stations are each separated by about $120^{\circ}$ in longitude, so at least one station is on the nightside at all times. We plan to analyze the real-time data at each station using the Pi 2 detection algorithm, and to exchange the detection results among these stations via the Internet. Therefore we can obtain information about substorm onsets in real-time, even if we are on the dayside. We have constructed a system to detect Pi 2 pulsations automatically at Mineyama observatory. The detection results for the period of February to August 1996 showed that the rate of successful detection of Pi 2 pulsations was $83.4 \%$ for the nightside (18-06MLT) and 26.5\% for the dayside (06-18MLT). The detection results near local midnight (20-02MLT) give the rate of successful detection of $93.2 \%$.
\end{abstract}

\section{Introduction}

Pi 2 pulsations are defined as geomagnetic field variations with a period of 40-150 seconds and an irregular waveform (Jacobs et al., 1964). This type of pulsation observed on the ground has been studied by many researchers since the 1950s. In most of these studies Pi 2 pulsations were selected by visual inspection. Takahashi et al. (1995) utilized wave power in the Pi 2 frequency band, which is calculated by applying the discrete Fourier transform, for selecting Pi 2 pulsations. We propose a method to detect Pi 2 pulsations using a new data analysis technique called wavelet analysis, which was described originally by exploration geophysicists (e.g., Goupillaud et al., 1984) and has been developed recently (e.g., Chui, 1992; Meyer, 1993; Chui et al., 1994; Kaiser, 1994; Wickerhauser, 1994). Several papers employing wavelet analysis appear in the field of magnetospheric physics (Ochadlick et al., 1993; Holter et al., 1995; Lui and Najmi, 1997). They adopted the continuous wavelet transform, but in this study we used the discrete wavelet transform which can be orthonormal. As wavelet analysis can express the time at which a particular signal appears, it is more suitable for investigating transient waves such as $\mathrm{Pi} 2$ pulsations. Using this method, we can make a list of Pi 2 pulsations which are identified objectively.

It is generally accepted that $\mathrm{Pi} 2$ pulsations are almost al-

Copy right (C) The Society of Geomagnetism and Earth, Planetary and Space Sciences (SGEPSS); The Seismological Society of Japan; The Volcanological Society of Japan; The Geodetic Society of Japan; The Japanese Society for Planetary Sciences. ways observed on the ground at substorm onsets and appear clearly in mid- or low-latitudes on the nightside (Saito et al., 1976a, b; Sakurai and Saito, 1976). Substorm onsets are also identified by other phenomena, for example, auroral breakups, sudden increases of the AE index, and mid-latitude positive bays. However, Pi 2 pulsations are more useful than these phenomena for substorm detection, because Pi 2 pulsations in mid- or low-latitudes are more sensitive to substorm onset (Saito et al., 1976b). Our method for detecting Pi 2 pulsations by wavelet analysis was applied for substorm monitoring. We aim at the nowcasting of substorm onsets, using real-time geomagnetic field data obtained at three stations. Saito et al. (1976a) have also proposed a "Pi 2 index" to monitor substorm activity and infer their characteristics which can be determined from the induction magnetograms obtained at three low-latitude stations with a mutual longitudinal separation of about $120^{\circ}$. Although our idea is similar to that by Saito et al. (1976a), recent progress in computers and instruments for data acquisition has allowed us to construct a substorm monitoring system in practice.

This paper is laid out as follows. In Section 2 we describe wavelet analysis mathematically. We show the validity of wavelet analysis for Pi 2 detection in Section 3. In Section 4 our project which aims at the nowcasting of substorm onsets is introduced; in addition, the automated $\mathrm{Pi} 2$ detection system at Mineyama observatory is explained and detection results are presented. 


\section{Orthonormal Wavelet Analysis \\ 2.1 Basics}

In Fourier analysis, a time series is decomposed into harmonic functions. Relations between a function $h(t)$ in the time domain and its Fourier transform $H(f)$ in the frequency domain are as follows,

$$
\begin{gathered}
h(t)=\int_{-\infty}^{\infty} H(f) e^{i 2 \pi f t} d f, \\
H(f)=\int_{-\infty}^{\infty} h(t)\left(e^{i 2 \pi f t}\right)^{*} d t,
\end{gathered}
$$

where the asterisk denotes the complex conjugate. The Fourier transform is utilized widely to analyze time series data, but it has a problem which comes from the characteristics of the harmonic functions. As the harmonic functions have finite values for $t \rightarrow \pm \infty$, Fourier analysis is sometimes not appropriate to analyze phenomena localized in time.

In wavelet analysis, a time series is decomposed into the basis functions (wavelets) which are localized in time and limited in a specified frequency range. Thus wavelet analysis is a suitable method for investigating the wave power of phenomena which are limited in both time and frequency, such as Pi 2 pulsations. The time series is mapped to the timefrequency domain, so the wavelet transform has two parameters which correspond to time and frequency. There are two types of wavelet transform, namely, the continuous transform and the discrete transform. The continuous wavelet transform is not orthonormal, but the discrete wavelet can construct an orthonormal set. For a time series $x(t)$, the discrete wavelet transform is expanded as

$$
\begin{aligned}
x(t) & =\sum_{j} \sum_{k} \alpha_{j, k} \psi_{j, k}(t), \\
\alpha_{j, k} & =\int_{-\infty}^{\infty} x(t) \psi_{j, k}^{*}(t) d t,
\end{aligned}
$$

where $\alpha_{j, k}$ is the wavelet coefficient and $\psi_{j, k}(t)$ is the discrete wavelet set. $\psi_{j, k}(t)$ is constructed from an analyzing wavelet $\psi(t)$, which generates the orthonormal discrete wavelet set, by

$$
\psi_{j, k}(t)=2^{\frac{j}{2}} \psi\left(2^{j} t-k\right),
$$

where $j$ and $k$ are integers. From Eq. (5) we can see that $j$ is related to the dilation of $\psi(t)$ and $k$ is related to the shift of $\psi(t)$ in the time domain. Thus $j$ and $k$ correspond to frequency and time, respectively.

\subsection{Meyer wavelet}

A number of analyzing wavelets to generate an orthonormal discrete wavelet set have been found. For example, the Haar wavelet, the Daubechies wavelet (Daubechies, 1988), and the Meyer wavelet (Meyer, 1989). It is reasonable to use the Meyer wavelet for analyzing time series data because the Meyer wavelet is band-limited in frequency. The Meyer wavelet is expressed as follows (Yamada and Ohkitani, 1991; Sasaki et al., 1992; Sato and Yamada, 1994; Yamanaka et al., 1994; Yomogida, 1994):

$$
\psi(t)=\frac{1}{2 \pi} \int_{-\infty}^{\infty} \Psi(\omega) e^{i \omega t} d \omega,
$$

where

$$
\Psi(\omega)=\left\{\begin{array}{cc}
0 & \left(|\omega| \geq \frac{8}{3} \pi\right) \\
e^{-i \frac{\omega}{2}}\left[1+\exp \left\{\frac{32}{3} \pi \frac{|\omega|-2 \pi}{\left(|\omega|-\frac{8}{3} \pi\right)^{2}\left(|\omega|-\frac{4}{3} \pi\right)^{2}}\right\}\right]^{-\frac{1}{2}} & \left(\frac{4}{3} \pi<|\omega|<\frac{8}{3} \pi\right) \\
e^{-i \frac{\omega}{2}} \quad\left(|\omega|=\frac{4}{3} \pi\right) & \\
e^{-i \frac{\omega}{2}}\left[1+\exp \left\{-\frac{4}{3} \pi \frac{|\omega|-\pi}{\left(|\omega|-\frac{4}{3} \pi\right)^{2}\left(|\omega|-\frac{2}{3} \pi\right)^{2}}\right\}\right]^{-\frac{1}{2}} & \left(\frac{2}{3} \pi<|\omega|<\frac{4}{3} \pi\right) \\
0 \quad\left(0 \leq|\omega| \leq \frac{2}{3} \pi\right)
\end{array} .\right.
$$

For actual analysis we use discrete time series and take a finite data length. Assuming a time series which has a sampling rate $\Delta t$ and a number of data points $N\left(N=2^{n}, n\right.$ is integer), we will obtain wavelet coefficients $\alpha_{j, k}$ confined in $0 \leq j \leq n-1$ and $0 \leq k \leq 2^{j}-1$. The frequency band for each $j$ is $2^{j} / 3 T \leq f \leq 2^{j+2} / 3 T$, where $T$ is the data length $(T=N \Delta t)$. Note that the width of time and frequency ranges covered by $\alpha_{j, k}$ are $T / 2^{j}$ and $2^{j} / T$, respectively. This implies that the wavelet coefficient with a large value of $j$ has high resolution in time and low resolution in frequency, and vice versa. The Nyquist frequency is included in the frequency range supported by the maximum value of $j$.

Figure 1 shows time plots of the wavelet functions $\psi_{j, k}(t)$ which were generated from the Meyer wavelet with a number of data points $N=1024=2^{10}$. Note that the Meyer wavelet has a symmetrical waveform. From Figs. 1(a)-1(c) which give examples of wavelet functions with different $j$ 's, we see that the smaller the value of $j$, the more the wavelet functions are dilated. Figures 1(d)-1(f) which give examples of wavelet functions with different $k$ 's show that the smaller the value of $k$, the earlier in time the non-zero part of the wavelet functions appear. Therefore we can discuss phenomena from the view point of both frequency $(j)$ and time $(k)$. Even if more than one wave packet which have the same frequency appear at different times, these phenomena are characterized by wavelet coefficients with different $k$ 's. This is one of the advantages of wavelet analysis.

\section{Detection of Pi 2 Pulsations by Wavelet Analysis 3.1 Example of wavelet analysis}

We have analyzed geomagnetic field data from Kakioka (26.9 $9^{\circ}$ geomagnetic latitude, $208.3^{\circ}$ geomagnetic longitude) using the Meyer wavelet. We took 512 data points of Kakioka data with a sampling rate of 1 second (i.e., data period of 512 seconds). Instead of tapering the time series by the data window function used in the Fourier analysis, we added 256 data points to each end of the data set; the value of the additional data is given to be equal to that of each end of the data set. This is because the tapered time series gives inappropriate values of wavelet coefficients around each end. The frequency range and time resolution of the wavelet functions for $j=3-9$ used in this study are shown in Table 1 . We note that Pi 2 pulsations which have a frequency range from 6.67 to $25.0 \mathrm{mHz}$ are mainly represented by wavelets with $j=4$ and 5. Examples of the wavelet analysis for the geomagnetic field data from Kakioka are shown in Fig. 2. The 

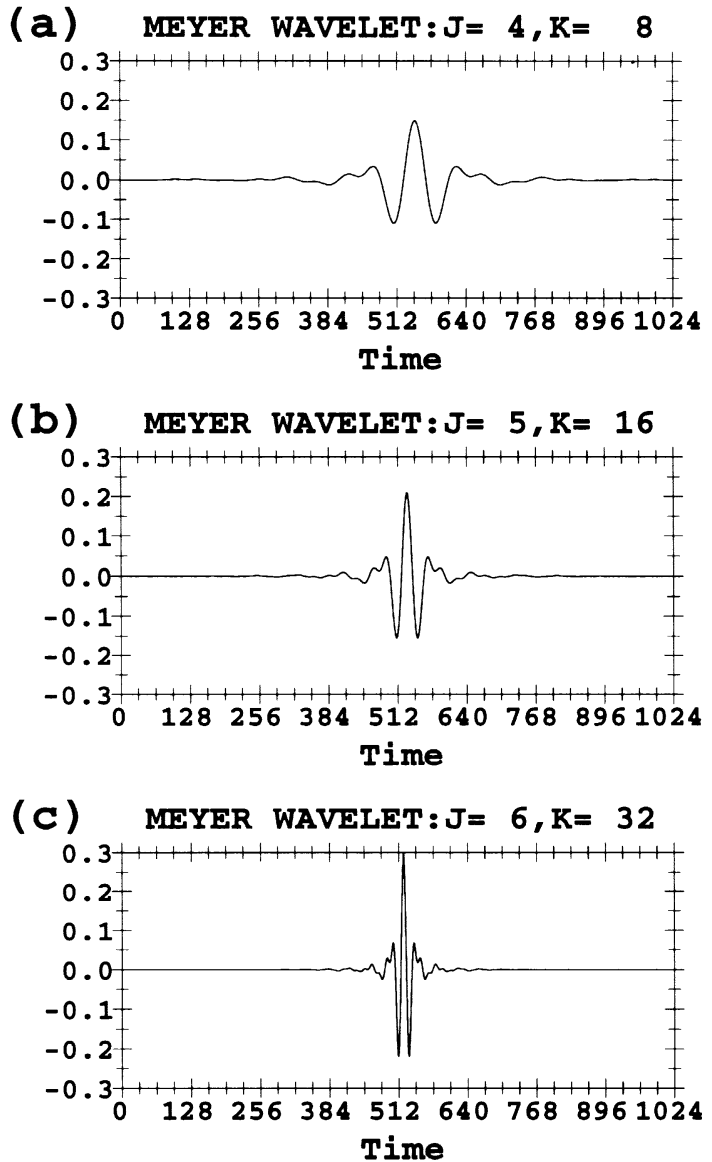

(d) MEYER WAVELET: $J=5, K=8$

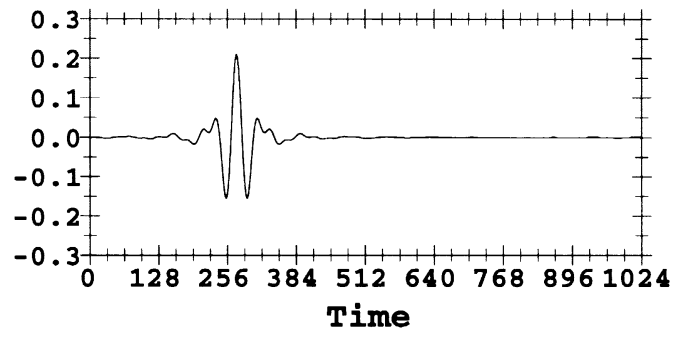

(e) MEYER WAVELET: $J=5, K=16$

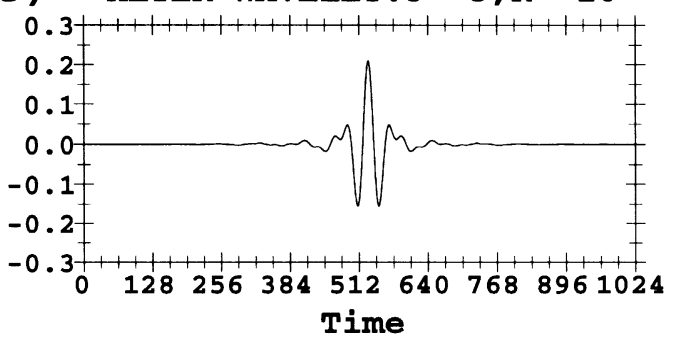

(f) MEYER WAVELET : $J=5, K=24$

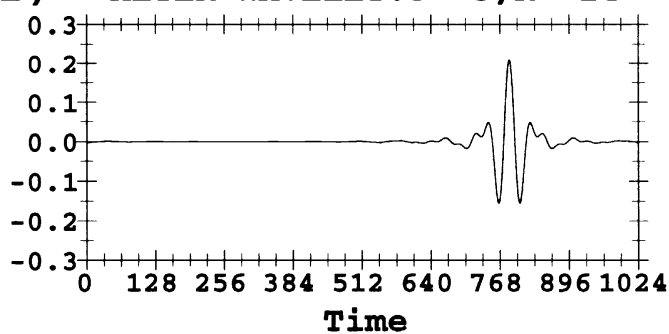

Fig. 1. Time plots of wavelet functions, which were generated from the Meyer wavelet, with $(j, k)$ of $(\mathrm{a})(4,8),(\mathrm{b})(5,16),(\mathrm{c})(6,32),(\mathrm{d})(5,8)$, (e) $(5,16)$, and (f) $(5,24)$.

left four panels of Figs. 2(a)-2(d) show the $H$-component and each panel shows data for the 512 second interval before the time indicated on the top of each panel. Thus it is noticed that 3 minutes have passed from Fig. 2(a) (1517UT) to Fig. 2(d) (1520UT). The right panels show normalized wavelet coefficients $\sqrt{2^{j} / T}\left|\alpha_{j, k}\right|$ for $j=4-6$ corresponding to the geomagnetic field data in the left panels. Scanning the right panels, we find wavelet coefficients for $j=5$ having large values of $0.8-1.2$ in Figs. 2(c) and 2(d), which are indicated by shading. It was found in the left panels of Figs. 2(c) and 2(d) that Pi 2 pulsations with a period of about 50 seconds $(f \sim 20 \mathrm{mHz})$ appeared at the same time that these large wavelet coefficients appeared. Therefore we can find Pi 2 pulsations by detecting large wavelet coefficients for $j=4$ or 5 . The criteria of detection (i.e., magnitude of wavelet coefficients, etc.) will be determined empirically.

In the left panels of Figs. 2(a)-2(d), we see that artificial noise with an amplitude of about $1 \mathrm{nT}$ appeared approximately 4 minutes before the onset time of the $\mathrm{Pi} 2$ pulsation. However, this artificial noise has no effect on the wavelet coefficients for $j=4$ and 5 , because it is too limited in time to be represented by wavelet functions for $j=4$ and 5 . Figure 3 shows the $H$-component of the geomagnetic field data, which is the same as that of Fig. 2(c), and corresponding normalized wavelet coefficients for $j=4-9$. The artificial noise in the left panel was represented by a large wavelet
Table 1. Frequency range and time resolution of the wavelet functions for $j=3-9$ used in this study.

\begin{tabular}{ccc}
\hline & $\begin{array}{c}\text { Frequency range } \\
(\mathrm{mHz})\end{array}$ & $\begin{array}{c}\text { Time resolution } \\
(\mathrm{sec} .)\end{array}$ \\
\hline 9 & $167-677$ & 2 \\
8 & $83.3-333$ & 4 \\
7 & $41.7-167$ & 8 \\
6 & $20.8-83.3$ & 16 \\
5 & $10.4-41.7$ & 32 \\
4 & $5.21-20.8$ & 64 \\
3 & $2.60-10.4$ & 128 \\
\hline
\end{tabular}

coefficient for $j=9$ which is indicated by an arrow. The Pi 2 pulsation which appeared around 1517UT is represented by a large wavelet coefficient for $j=5$ and did not affect wavelet coefficients for $j=9$. Thus we can distinguish spiky noise by checking the wavelet coefficients for large values of $j$. In the case of Fourier analysis, it is difficult to distinguish artificial noise from other phenomena, because artificial noise like spikes affects the spectral powers in all 

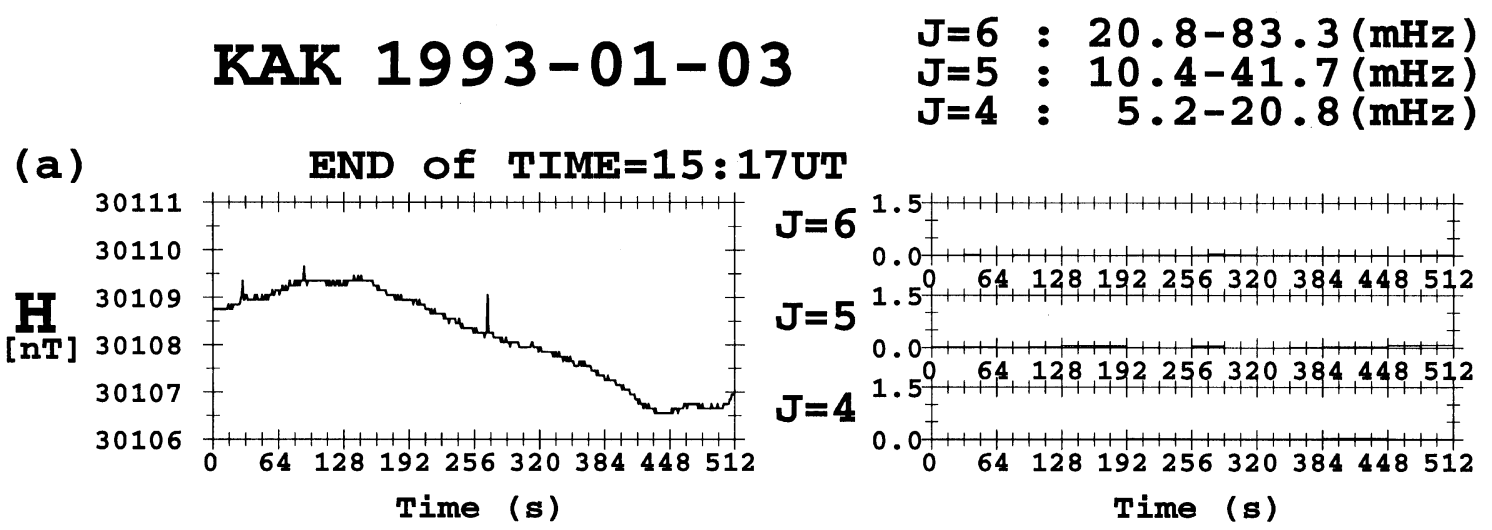

(b) END of TIME=15:18UT

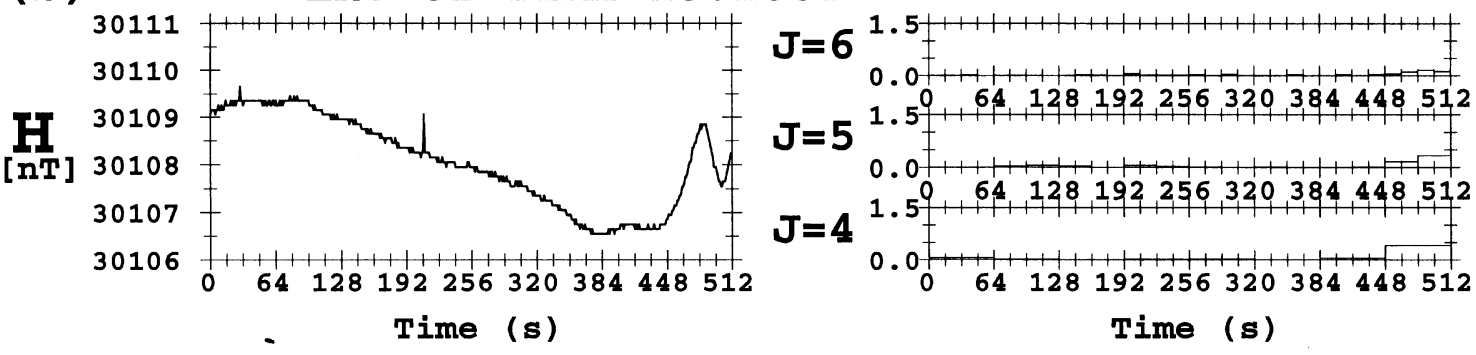

(c) END of TIME=15:19UT

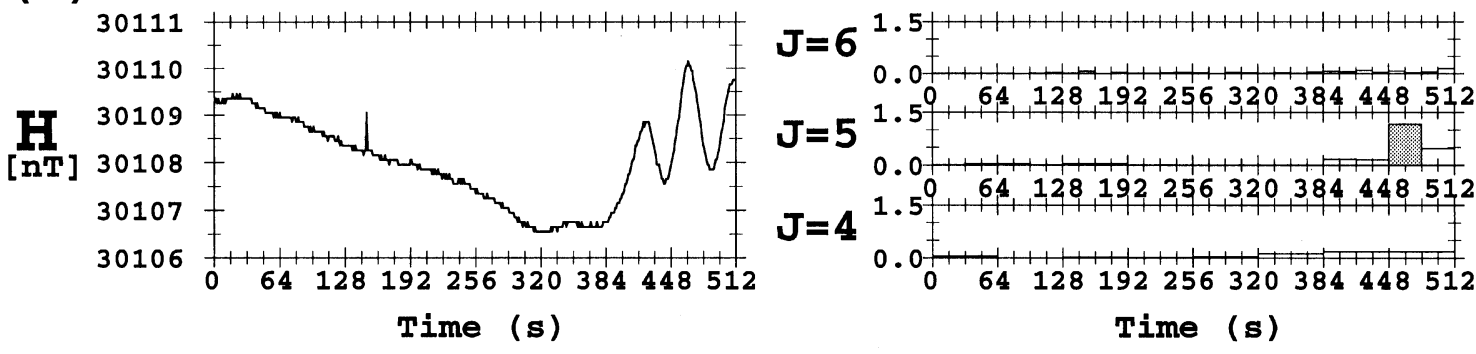

(d) END of TIME=15:20UT

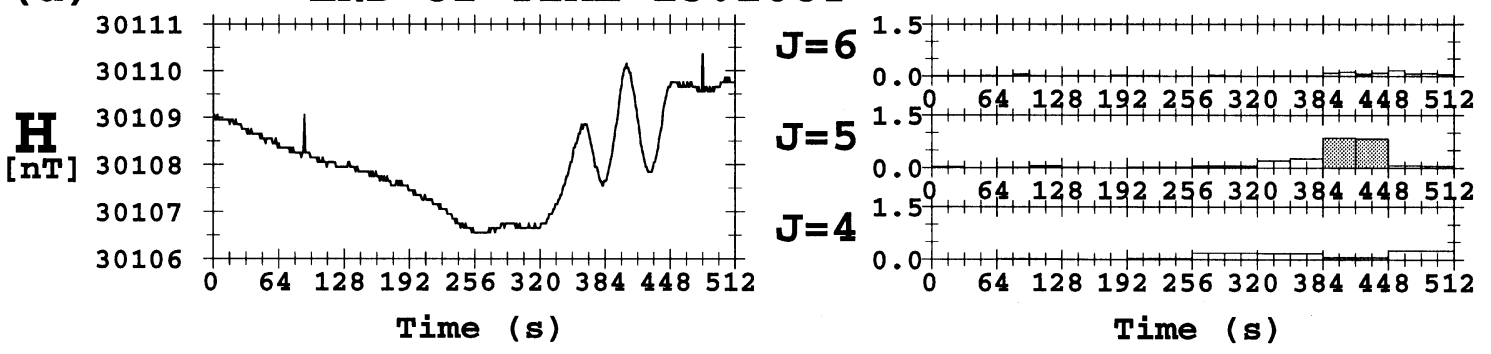

Fig. 2. Examples of wavelet analysis for the geomagnetic field data. (a) The left panel shows the $H$-component of the geomagnetic field data from Kakioka for the interval 512 seconds before 1517UT. The right panel shows wavelet coefficients for $j=4-6$ corresponding to the geomagnetic field data in the left panel. (b)-(d) Same as Fig. 2(a) except for the end of time of 1518UT, 1519UT, and 1520UT, respectively.

frequency ranges. Wavelet analysis has the advantage that spiky noise can be distinguished easily from Pi 2 pulsations. 3.2 Algorithm for $\mathbf{P i} 2$ detection

We have developed an algorithm to detect Pi 2 pulsations automatically. The algorithm consists of procedures to analyze the $H$ - and $D$-components of the geomagnetic field data every one minute using the Meyer wavelet and to detect wavelet coefficients for $j=4$ or 5 which exceed the criteria of detection. We tested whether the algorithm can detect
Pi 2 pulsations properly, using Kakioka data on the nightside (from 18LT through midnight to 06LT) in the period of January 1993. The results of the algorithm detection were compared with those of visual detection.

3.2.1 Comparison between the algorithm detection and the visual detection (Amplitude) Table 2 shows the number of events detected by these two methods. Events detected as $\mathrm{Pi} 2$ pulsations by the algorithm were classified into four categories (i.e., Quality-AA, -A, -B, and -C) according to 


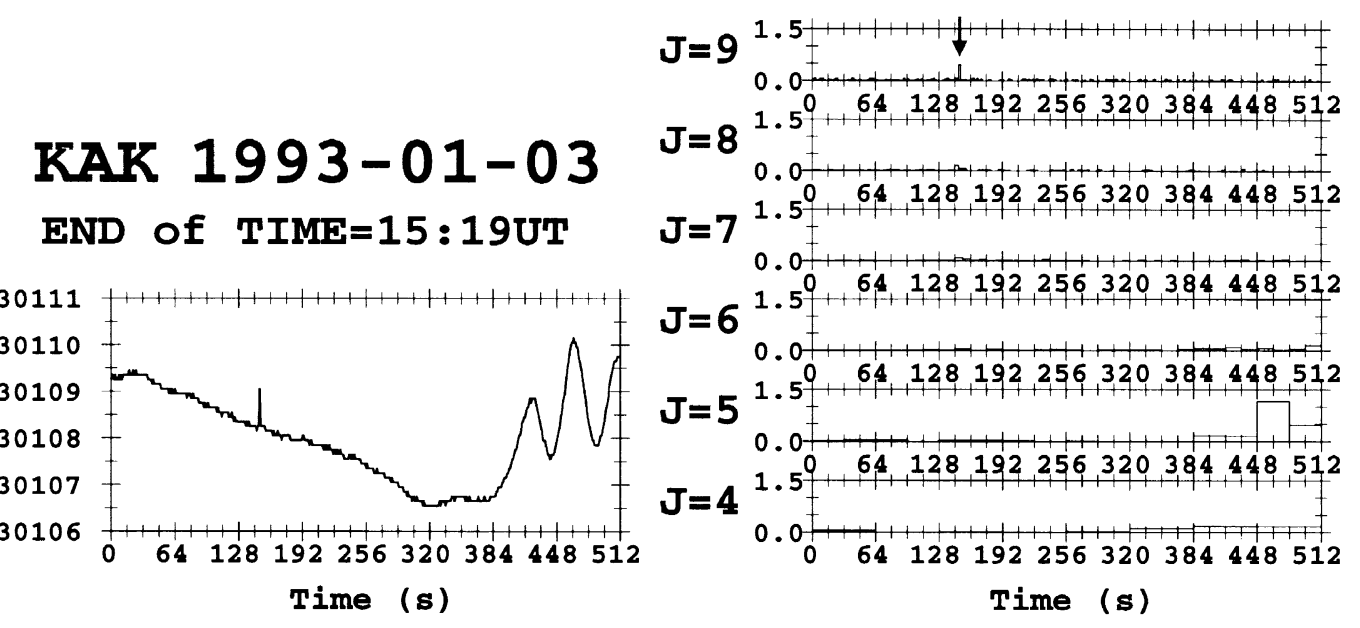

Fig. 3. The $H$-component of the geomagnetic field data, which is the same as that of Fig. 2(c), and corresponding wavelet coefficients for $j=4-9$.

Table 2. The number of events detected by the wavelet analysis and visual inspection from Kakioka data on the nightside (18-06LT) during January 1993.

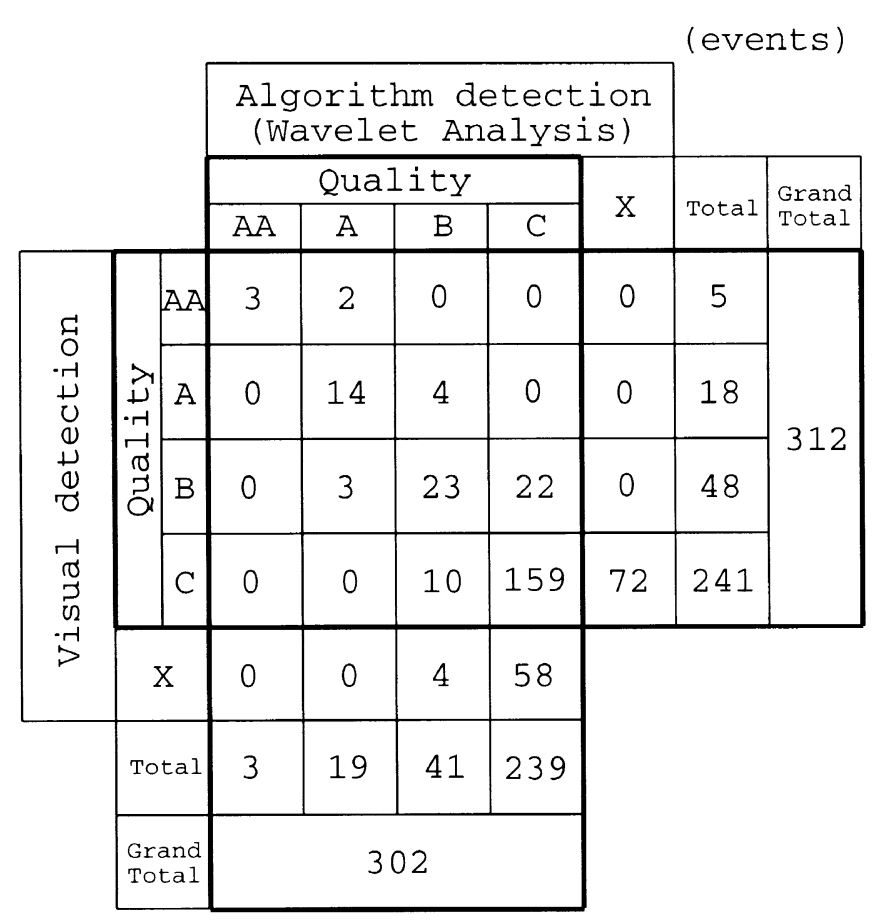

peak-to-peak amplitudes of events. The peak-to-peak amplitudes were estimated from the value of the wavelet coefficient by multiplying by a given constant which is for converting the wavelet coefficient to the wavelet amplitude. In the case that the wavelet coefficients in both the $H$ - and $D$-components exceed the criteria, we calculated the sum of the square of estimated amplitudes in the $H$ - and $D$-components and took the square root of the sum as the peak-to-peak amplitude of the event. Quality-AA represents events whose peak-to-peak amplitudes are more than $3.0 \mathrm{nT}$. Quality-A, -B, and -C are for events which have peak-to-peak amplitudes from $1.8 \mathrm{nT}$ up to $3.0 \mathrm{nT}$, from $1.2 \mathrm{nT}$ up to $1.8 \mathrm{nT}$, and from $0.6 \mathrm{nT}$ up to $1.2 \mathrm{nT}$, respectively. The detection algorithm neglects events whose peak-to-peak amplitudes given by wavelet coefficients are less than $0.6 \mathrm{nT}$. The onset time of the event is determined from the value of $k$ of the wavelet coefficient which exceeds the criteria. We also scanned visually the plots of the raw and bandpass filtered magnetic field data to detect Pi 2 pulsations. The period range of the bandpass filter is from 40 to 150 seconds. Pi 2 pulsations detected visually were then classified into the same four categories as those for the algorithm detection, according to peak-to-peak amplitudes which were determined from the bandpass filtered magnetic field data. The onset time of Pi 2 pulsation by the visual detection was determined as the time when a sudden change of the geomagnetic field followed by damped oscillations appeared. The class denoted by $\mathrm{X}$ in Table 2 indicates events which were detected by one detection method but were not 
(a)

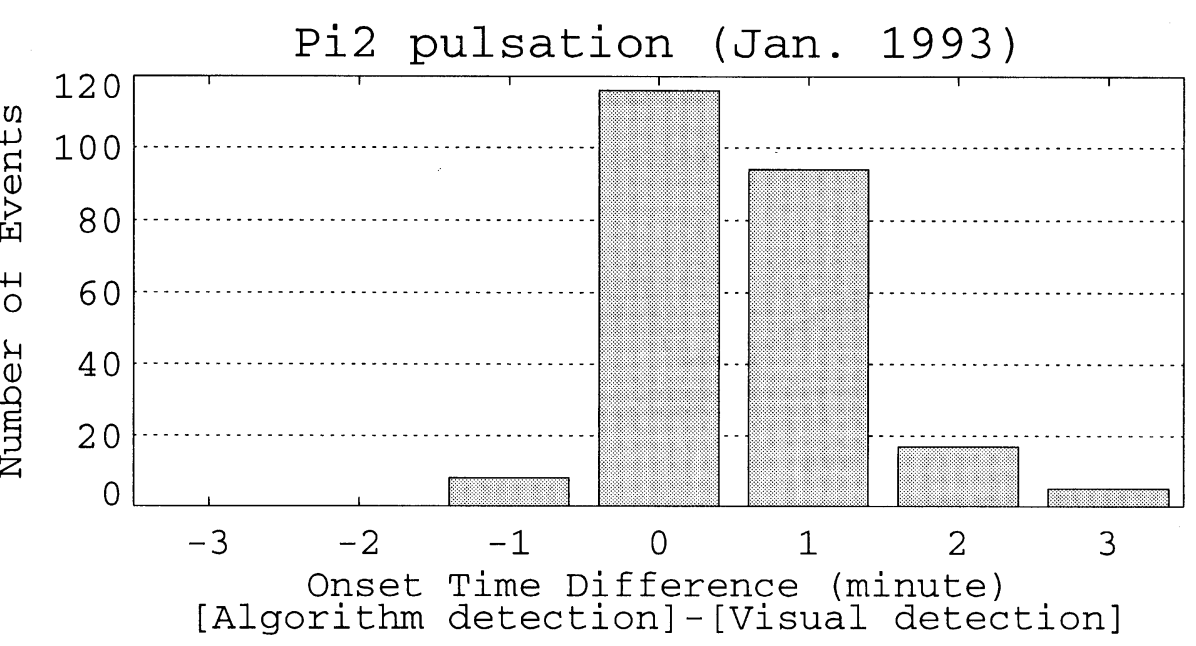

(b)

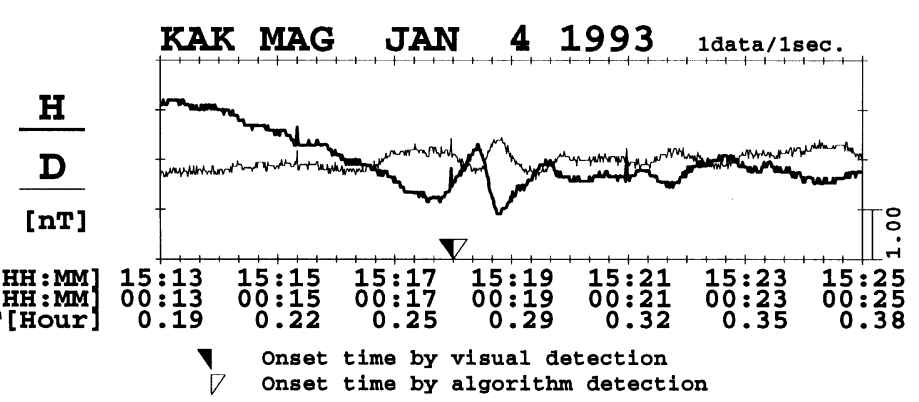

(c)

KAK MAG JAN 27,1993 1data/1sec.

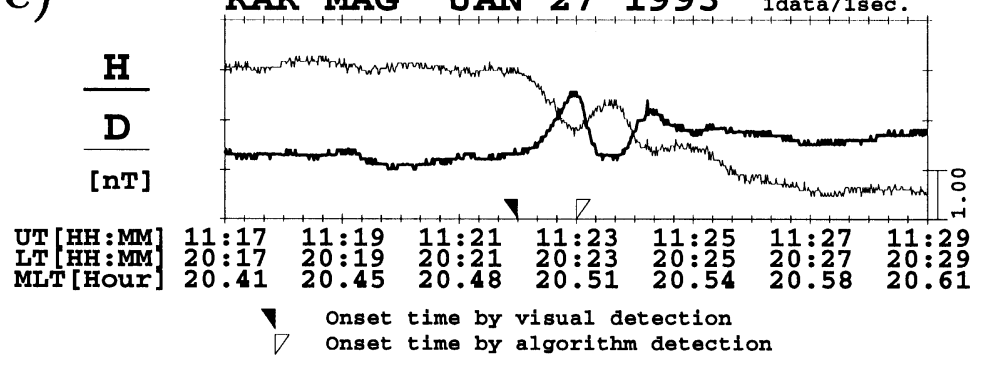

(d)

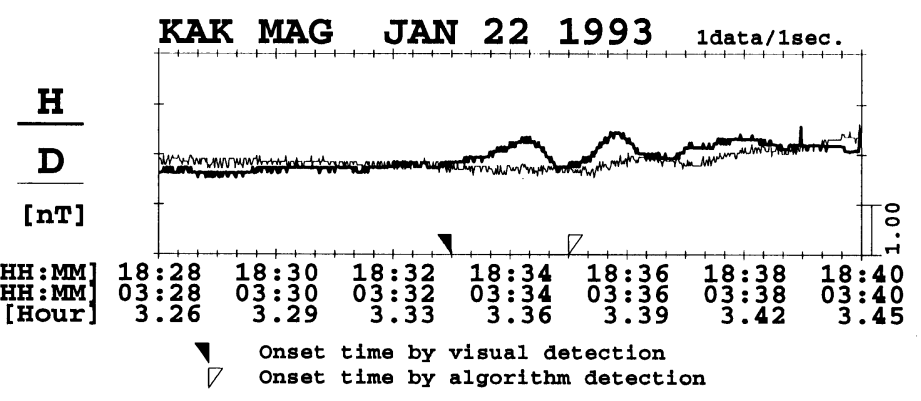

Fig. 4. (a) Distribution of the onset time difference, which is defined as the onset time determined by the algorithm detection minus that by the visual detection. (b)-(d) Examples of Pi 2 pulsations with onset time differences of 0, 1, and 2 minute(s), respectively. 


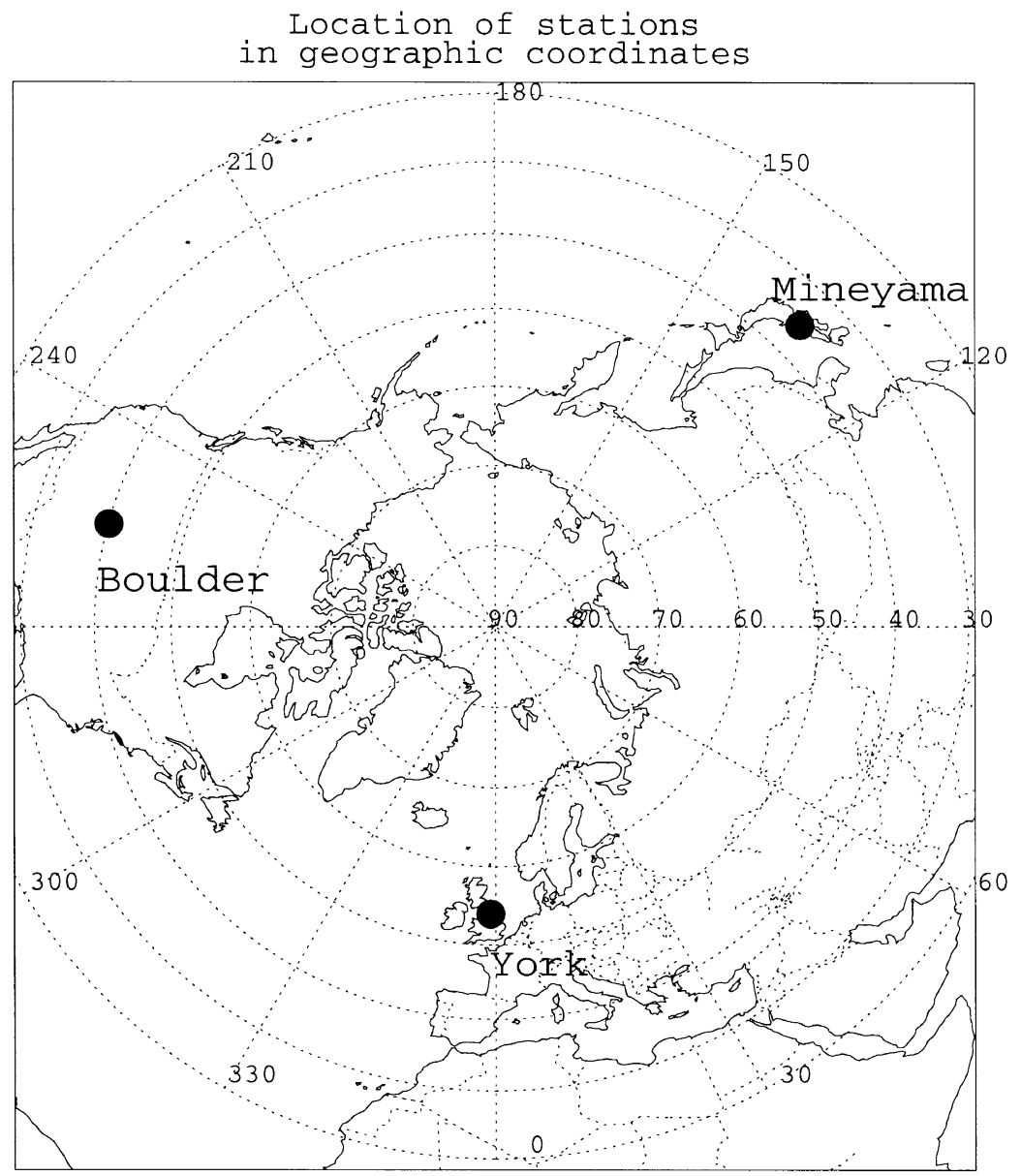

Fig. 5. Map showing the locations of the three stations (Mineyama in Japan, the York SAMNET station in the U.K., and Boulder in the U.S.) in geographic coordinates. The three stations are each separated by about $120^{\circ}$ in longitude.

detected by the other method. If the difference between the onset time determined by the algorithm and that by the visual detection was less than 3 minutes, we considered that both detection methods found the same event. Here a maximum threshold of time difference of 3 minutes was allowed, because we expected that there may be a few minute ambiguity in some events from our experience.

We note that 3 Quality-AA events found by wavelet analysis were also detected by the visual inspection as Pi 2 pulsations in Quality-AA. Out of 19 Quality-A events from the algorithm detection, 14 events were also detected visually as Pi 2 pulsations in Quality-A, and 5 events were found by the visual inspection as Pi 2 pulsations in other categories (Quality-AA and -B). Twenty-three events were recognized as Quality-B by both the algorithm and the visual inspection. Fourteen events were found as Quality-B events by the algorithm and as Quality-A or -C pulsations by the visual inspection. There were 4 events which were detected as Quality-B events by the algorithm but were not found by the visual inspection. We note that out of 239 Quality-C events from the algorithm detection, 159 events were detected visually in the same category (Quality-C) and 22 events were detected visually in the different category (Quality-B), but 58 events were not found by the visual inspection. From Table 2 we conclude that out of 302 events detected by the wavelet analysis, 240 events (79.5\%) were detected as Pi 2 pulsations by the visual inspection, and 199 events $(65.9 \%)$ were detected visually as Pi 2 pulsations in the same categories as those by the wavelet analysis.

A detailed examination of the 62 events which were not detected by the visual inspection showed that 10 of the events were actual $\mathrm{Pi} 2$ pulsations and 30 of the events were different phenomena from Pi 2 pulsations. It is thought that the visual inspection method failed to detect these 10 events of Pi 2 pulsations. With respect to the rest of the events (22 events), although the onset time of a Pi 2 pulsation was detected properly, the detection algorithm reported occurrence of another event while that Pi 2 pulsations was decaying. Thus the rate of successful detection by this algorithm, which is defined as the ratio of the number of Pi 2 pulsations detected successfully to the number of the events detected by the wavelet analysis, is calculated to be $82.8 \%((240+10) / 302)$.

It should be noted that there were $72 \mathrm{Pi} 2$ pulsations in Quality-C which were not detected by the detection algorithm. These pulsations are thought to have peak-to-peak amplitudes close to $0.6 \mathrm{nT}$ which is the minimum threshold of Pi 2 detection for the algorithm.

3.2.2 Comparison between the algorithm detection and the visual detection (onset time) From Kakioka data on the nightside (18-06LT) during January 1993, 240 Pi 2 
Table 3. Coordinates of the three stations (Mineyama in Japan, the York SAMNET station in the U.K., and Boulder in the U.S.).

\begin{tabular}{cccccc}
\hline \multirow{2}{*}{ Station } & \multicolumn{2}{c}{ Geographic } & & \multicolumn{2}{c}{ Geomagnetic } \\
\cline { 2 - 3 } \cline { 5 - 6 } \cline { 5 - 6 } Mineyama & Latitude $\left({ }^{\circ} \mathrm{N}\right)$ & Longitude $\left({ }^{\circ} \mathrm{E}\right)$ & & Latitude $\left({ }^{\circ} \mathrm{N}\right)$ & Longitude $\left({ }^{\circ} \mathrm{E}\right)$ \\
York & 35.6 & 135.1 & & 25.5 & 203.2 \\
Boulder & 54.0 & 359.0 & & 51.0 & 78.6 \\
\hline
\end{tabular}

pulsations were detected by both the algorithm and the visual inspection, as indicated in Table 2. We investigated the difference between the onset time determined by the algorithm detection and that by the visual detection for these 240 events. The result is presented in Fig. 4(a). The onset time difference was defined as the onset time determined by the algorithm detection minus that by the visual detection. A positive value of the onset time difference means that the onset time by the algorithm detection lags behind that by the visual detection. Figure 4(a) shows that the onset time by the algorithm detection is the same as or 1 minute later than that by the visual detection in most of the events. Figures 4(b) and 4(c) give typical examples of these events. Figure 4(a) also shows that there are much more events in the bins of positive time difference than those of negative time difference. Thus we can say that the onset time determined by the algorithm detection tends to lag behind that by the visual detection. We suppose these results are due to the way of Pi 2 detection by the algorithm, that is, detecting a large wavelet coefficient as mentioned above. A large wavelet coefficient corresponds to a peak in oscillation of the geomagnetic field. Thus the onset time determined by the algorithm detection is close to the time of the first peak of a $\mathrm{Pi} 2$ pulsation which lags behind the beginning of a Pi 2 pulsation.

There are some events in the bins of time difference larger than 2 minutes. These events are thought to start oscillating with a small amplitude and then develop amplitudes larger than $0.6 \mathrm{nT}$ a few minutes later. Figure 4(d) shows an example of the onset time difference of 2 minutes. Notice that the peak-to-peak amplitude of the Pi 2 pulsation in Fig. 4(d) was about $0.5 \mathrm{nT}$ at first and increased to about $0.7 \mathrm{nT}$ later.

The average of the onset time difference is calculated to be +0.56 minutes $(+34$ seconds). We found that in $90.8 \%$ $(97.9 \%)$ of the events the onset time determined by the algorithm detection coincides with that by the visual detection with an accuracy of \pm 1 minute ( \pm 2 minutes). The results of onset time should be used carefully, if one needs to know the onset time of Pi 2 pulsation with an accuracy less than 1-2 minutes.

\section{Application for Nowcasting of Substorm Onsets 4.1 Outline of project}

We have a project which aims at the nowcasting of substorm onsets by detecting Pi 2 pulsations in real-time using wavelet analysis. We use real-time geomagnetic field data, with a sampling rate of 1 second, obtained at mid- and lowlatitude stations (Mineyama in Japan, the York SAMNET station in the U.K., and Boulder in the U.S.) shown in Fig. 5.

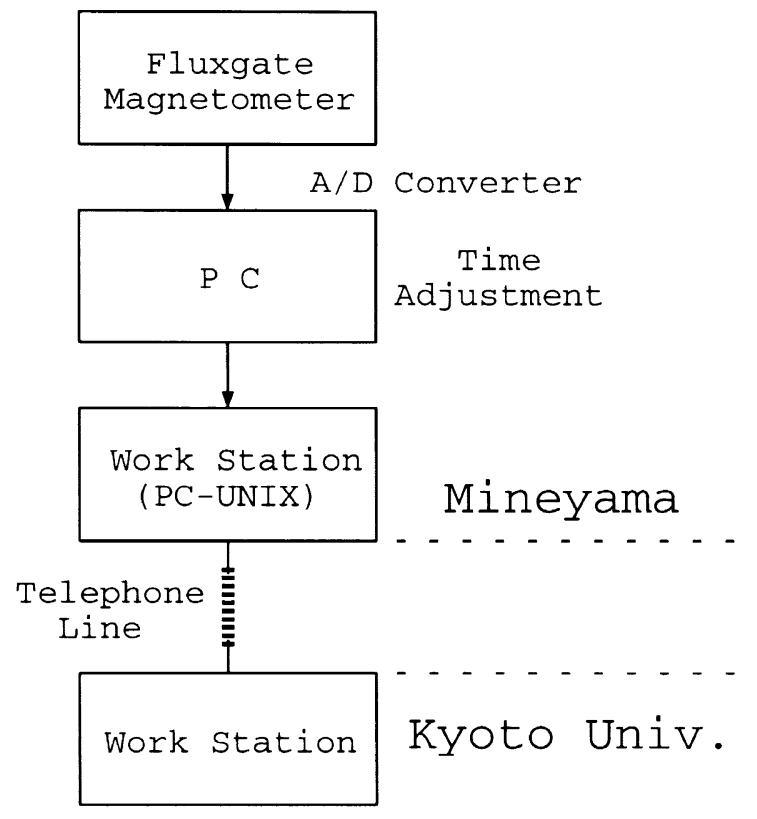

Fig. 6. Schematic diagram of the automated Pi 2 detection system at Mineyama.

The locations of the three stations are listed in Table 3. These stations are each separated by about $120^{\circ}$ in longitude, so at least one station is on the nightside at all times. The realtime data are analyzed by essentially the same detection algorithm as described in the previous section, and the detection results will be exchanged among these stations via the Internet. Therefore we can obtain information about substorm onsets in real-time, even if we are on the dayside.

\subsection{Operation at Mineyama observatory}

4.2.1 Pi 2 detection system We have constructed a system for observation of the geomagnetic field data at Mineyama, which is about $100 \mathrm{~km}$ northwest of Kyoto. The data are obtained by a fluxgate magnetometer with a sampling rate of 1 second. We have also constructed both hardware and software systems to detect Pi 2 pulsations automatically for monitoring substorm onsets (Fig. 6). The data obtained by the fluxgate magnetometer are sent to a UNIX work station at Mineyama observatory every minute through a PC, in which a time adjustment is carried out. The geomagnetic field data are analyzed immediately by the software installed in the work station, which contains the algorithm to detect Pi 2 pulsations. If a Pi 2 pulsation is detected, information about the Pi 2 pulsation is transmitted to a work station at 

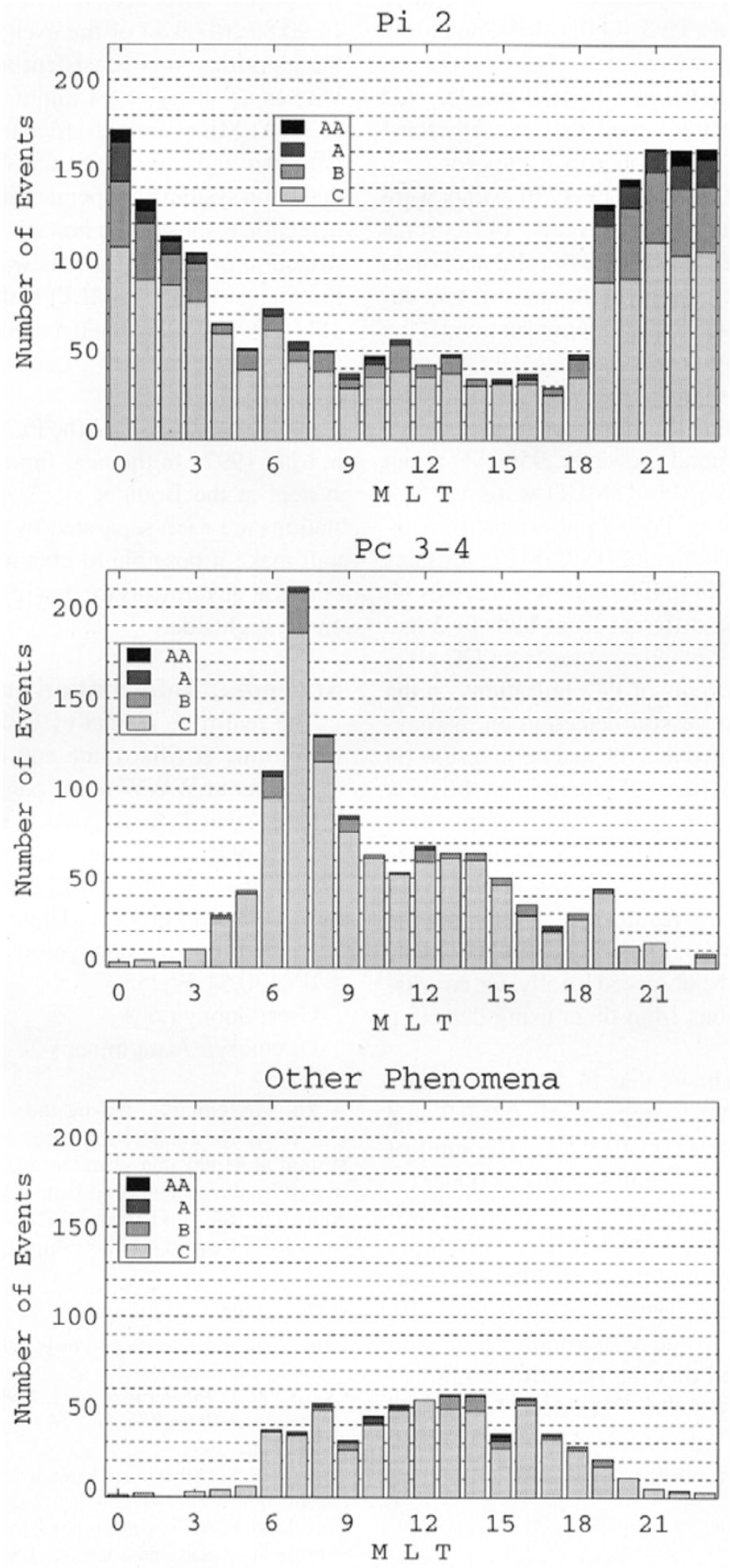

Fig. 7. MLT dependence of the number of events detected at Mineyama, which were classified into three categories according to visual inspection of waveforms. (top) Pi 2 pulsations, (middle) Pc 3-4 pulsations, and (bottom) other phenomena.

Kyoto University down a telephone line by UUCP (Unix to Unix copy). We can obtain the information about Pi 2 pulsations within 3 minutes of the onset time for almost all the events. The detection system has been operating since February 1996. The results of Pi 2 pulsation detection can be found on our WWW homepage (see Appendix).
4.2.2 Detection results Using the results of detection at Mineyama for the period from February through August 1996, we examined the validity of the detection results. The detection system sometimes fails to distinguish between $\mathrm{Pi} 2$ pulsations and phenomena with almost the same frequency range such as Pc 3-4 pulsations. Thus the events detected by 
this system were checked visually and classified into three categories (i.e., Pi 2 pulsations, Pc 3-4 pulsations, and other phenomena) according to the waveforms. The events classified into Pi 2 pulsations are thought to be those detected properly by this system. Figure 7 gives the magnetic local time (MLT) dependence of the number of events for each category. On the nightside (18-06MLT), 1736 events were detected by this system. Out of 1736 events, 1447 events were Pi 2 pulsations and the rate of successful detection is calculated to be $83.4 \%$, which is almost the same as that derived from Kakioka data shown in the previous section. If the detection results near local midnight (20-02MLT) are considered, the rate of successful detection has a higher value of $93.2 \%$. Thus we confirm that this detection system works well on the nightside. We found, however, 956 events out of 2043 events on the dayside (06-18MLT) were not Pi 2 pulsations but Pc 3-4 pulsations. Pc 3-4 pulsations were detected frequently in the MLT range of 06-09MLT. Besides these Pc 3-4 pulsations, 26.7\% (545/2043) of the events on the dayside were phenomena different from both Pi 2 and Pc 3-4, or artificial noise that mainly comes from DC electrified train. We found 542 events of Pi 2 pulsations on the dayside, hence the rate of successful detection on the dayside is $26.5 \%$. It should be noted that the detection results on the dayside contain a large number of phenomena different from Pi 2 pulsations, especially in the MLT range of 0609MLT. The low rate of successful detection on the dayside will be improved by installing similar systems at both York and Boulder and comparing the results of detection among these three sites. As Pc 3-4 pulsations and other phenomena on the dayside are thought to be observed locally, we can distinguish dayside Pi 2 pulsations from them using detection results at York and Boulder.

The top panel of Fig. 7 shows that Pi 2 pulsations are observed frequently in the MLT range of 19-02MLT and have an occurrence peak around 00-01MLT. The ratio of the number of Pi 2 pulsations with large amplitudes (QualityAA, -A, and -B) to the number of all Pi 2 pulsations in each MLT bin is high in the MLT range of 19-02MLT. This shows that Pi 2 pulsations with large amplitudes are likely to occur around local midnight. These results are consistent with those by previous studies (Saito and Matsushita, 1968; Saito et al., 1976a). We also found that the ratio of the number of dayside Pi 2 pulsations (18-06MLT) to that of nightside Pi 2 pulsations (06-18MLT) was 37.5\% (542/1447), which means that Pi 2 pulsations are observed commonly even on the dayside.

\section{Conclusions}

In this paper we described a method for automated detection of Pi 2 pulsations and introduced our project which aims at the nowcasting of substorm onsets. We can summarize as follows.

1. For detecting Pi 2 pulsations in real-time we applied wavelet analysis which is a suitable method to investigate waves which are limited in both time and frequency. The Meyer wavelet was adopted as the analyzing wavelet.

2. An algorithm to detect Pi 2 pulsations automatically by wavelet analysis was developed. We tested this algorithm using Kakioka data on the nightside and found that (a) The rate of successful detection of Pi 2 pulsations was $82.8 \%$; (b) In $90.8 \%(97.9 \%)$ of the events the onset time reported by the algorithm was consistent with that determined visually with an accuracy of \pm 1 minute ( \pm 2 minutes).

3. At Mineyama observatory, we have constructed both hardware and software systems to detect Pi 2 pulsations. The detection system has operated since February 1996. From the detection results of the first six months, the rate of successful detection of Pi 2 pulsations was calculated to be $83.4 \%$ for the nightside (18-06MLT) and $26.5 \%$ for the dayside (06$18 \mathrm{MLT})$. If we use the detection results near local midnight (20-02MLT), the rate of successful detection increases to $93.2 \%$.

We started to operate the Pi 2 detection system at York site in May 1997. In the near future we plan to install a similar system at the Boulder site. As Mineyama and these two stations are each separated by about $120^{\circ}$ in longitude, this will make it possible to obtain information about substorm onsets at any time as there will be always at least one station on the nightside.

\section{Appendix. Information on Pi 2 Pulsations}

The real-time results of Pi 2 detection (onset times and waveforms at Mineyama and onset times at York) can be found on our WWW home page.

http://swdcdb.kugi.kyoto-u.ac.jp/

Daily plots of 1-minute Mineyama data and Pi 2 waveform plots with onset times of Pi 2 pulsations are generated in the form of PostScript files. These PostScript files are available from the following anonymous ftp directory.

IP: 130.54 .59 .253

User: anonymous

Directory: ./data/mineps

Acknowledgments. We are indebted to the staff at DPRI, Kyoto University for cooperating with us in operating the Pi 2 detection system at Mineyama observatory. This study has been supported in part by the special fund from Kyoto University for research and education, the fund from DPRI for Project Research, and grants from JSPS Research Fellowships for Young Scientists.

\section{References}

Chui, C. K., An Introduction to Wavelets, 266 pp., Academic Press, San Diego, California, 1992.

Chui, C. K., L. Montefusco, and L. Puccio (Eds.), Wavelets: Theory, Algorithms, and Applications, 627 pp., Academic Press, San Diego, California, 1994.

Daubechies, I., Orthonormal bases of compactly supported wavelets, Comm. Pure Appl. Math., 41, 909-996, 1988.

Goupillaud, P., A. Grossmann, and J. Morlet, Cycle-octave and related transforms in seismic signal analysis, Geoexploration, 23, 85-102, 1984.

Holter, Ø., C. Altman, A. Roux, S. Perraut, A. Pederson, H. Pécseli, B Lybekk, J. Trulsen, A. Korth, and G. Kremser, Characterization of low frequency oscillations at substorm breakup, J. Geophys. Res., 100, 1910919119, 1995.

Jacobs, J. A., Y. Kato, S. Matsushita, and V. A. Troitskaya, Classification of geomagnetic micropulsations, J. Geophys. Res., 69, 180-181, 1964.

Kaiser, G., A Friendly Guide to Wavelets, 300 pp., Birkhäuser, Boston, Massachusetts, 1994.

Lui, A. T. Y. and A.-H. Najmi, Time-frequency decomposition of signals in a current disruption event, Geophys. Res. Lett., 24, 3157-3160, 1997.

Meyer, Y., Orthonormal wavelets, in Wavelets, edited by J. M. Combes, A. Grossmann, and Ph. Tchamitchian, pp. 21-37, Springer-Verlag, Berlin, 1989.

Meyer, Y., Wavelets: Algorithms and Applications, translated by R. D. Ryan, 133 pp., Society for Industrial and Applied Mathematics, Philadelphia, 
Pennsylvania, 1993

Ochadlick, A. R., Jr., H. N. Kritikos, and R. Giegengack, Variations in the period of the sunspot cycle, Geophys. Res. Lett., 20, 1471-1474, 1993.

Saito, T. and S. Matsushita, Solar cycle effects on geomagnetic Pi 2 pulsations, J. Geophys. Res., 73, 267-286, 1968.

Saito, T., T. Sakurai, and Y. Koyama, Mechanism of association between Pi 2 pulsation and magnetospheric substorm, J. Atmos. Terr. Phys., 38, 1265-1277, 1976a.

Saito, T., K. Yumoto, and Y. Koyama, Magnetic pulsation Pi 2 as a sensitive indicator of magnetospheric substorm, Planet. Space Sci., 24, 1025-1029, 1976b.

Sakurai, T. and T. Saito, Magnetic pulsation Pi 2 and substorm onset, Planet. Space Sci., 24, 573-575, 1976.

Sasaki, F., T. Maeda, and M. Yamada, Study of time history data using wavelet transform, J. Struc. Eng. Architec. Inst. Japan, 38B, 9-20, 1992 (in Japanese with English abstract).

Sato, K. and M. Yamada, Vertical structure of atmospheric gravity waves revealed by the wavelet analysis, J. Geophys. Res., 99, 20623-20631,
1994

Takahashi, K., S. Ohtani, and B. J. Anderson, Statistical analysis of Pi 2 pulsations observed by the AMPTE CCE spacecraft in the inner magnetosphere, J. Geophys. Res., 100, 21929-21941, 1995.

Wickerhauser, M. V., Adapted Wavelet Analysis from Theory to Software, 486 pp., A. K. Peters, Wellesley, Massachusetts, 1994.

Yamada, M. and K. Ohkitani, Orthonormal wavelet analysis of turbulence, Fluid Dyn. Res., 8, 101-115, 1991.

Yamanaka, M. D., T. Shimomai, and S. Fukao, A model of quasi-monochromatic field of middle-atmospheric internal gravity waves, Proc. of the 1992 STEP Symposium/5th COSPAR Colloquium, 511-518, 1992.

Yomogida, K., Detection of anomalous seismic phases by the wavelet transform, Geophys. J. Int., 116, 119-130, 1994.

M. Nosé (e-mail: nose@kugi.kyoto-u.ac.jp), T. Iyemori, M. Takeda, T. Kamei, D. K. Milling, D. Orr, H. J. Singer, E. W. Worthington, and N. Sumitomo 